\title{
Diagnosis and Treatment of Status Epilepticus in a Pediatric Renal Recipient
}

\author{
Gao Hong-jun, Luo xiangdong, Liang Taisheng, Liang Fangfang et al. \\ Department of Transfusion Research, The Rui Kang Affiliated Hospital of Guangxi \\ Traditional Chinese Medical University, Nanning, \\ China
}

\section{Introduction}

Organ transplant traces its origins to a landmark operation by Murray in Boston in 1954 in which an identical twin received a donated sibling kidney. Since that seminal procedure, advances in tissue matching, improvements in surgical technique, and new and morepowerful immunosuppressive agents have increased the number and types of transplants that can be done. Advances in immunology and transplant technique have allowed longer survival for transplant recipients, but this has resulted in the emergence of several neurologic problems.

The kidney is the most frequently transplanted organ with more than 10000 transplants occurring per year worldwide. Since that historic operation, kidney transplants have developed into the best-accepted therapy for most causes of end-stage renal failure. The 1year survival rate is close to $100 \%$, with an $85 \%$ to $95 \%$ graft survival rate. However, despite these advances, neurologic complications after renal transplants occur approximately $30 \%$ of the time. Several characteristics of renal transplant patients make overall complication rates in these persons different from those of other organ transplant recipients. Many renal transplant patients have some degree of vascular compromise either as a result of their underlying disease (eg, hypertension, diabetes) or because of emboli associated with underlying atherosclerosis or heart disease. After transplant, neurologic complications may develop secondary to the transplant itself, the immunosuppressive agent, or a previously known organ parenchymal failure.Under the effect of immunosuppression and other factors, renal transplant recipients are likely to be afflicted with epilepsy, the attack rate of which can reach almost $20 \%$ among pediatric patients. However, at present there are insufficient statistics to explain the cause of this danger. ${ }^{1}$ Status epilepticus, also called grand mal seizures, can have a serious effect on the prognosis of renal transplant recipients. In particular, the first few attacks after kidney transplantation may have a serious effect on renal transplant recipients.

\section{Briefing of case history}

\subsection{Object}

A 17-year-old female patient was hospitalized because of dizziness and fatigue of more than 2 months' duration and with no obvious precipitating factor. Without timely treatment, she 
soon experienced syncope. She was hospitalized and examined, judged to have high blood pressure and a serum creatinine (SCr) level of $1374.9 \mu \mathrm{mmol} / \mathrm{L}$, diagnosed with chronic glomerulonephritis and uremia, and treated with hemodialysis.

\subsection{Preoperative diagnosis and treatment}

After being hospitalized, the patient was treated with regular hemodialysis and underwent a series of preoperative therapeutic measures including blood pressure control, anemia improvement, and weight control. On the 40th preoperative day, the patient developed acute loss of consciousness, expressionless eyes, foaming at the mouth, and convulsion of the limbs, all of which lasted for about $5 \mathrm{~min}$. Cerebral MRI was performed, but no abnormalities were detected. The patient was then diagnosed with symptomatic epilepsy. Carbamazepine was initially administered at a dosage of $0.1 \mathrm{~g}$ twice a day; the concentration was checked at regular intervals to adjust the dosage. After 2 weeks, the patient was treated with maintenance therapy at a dosage of $0.1 \mathrm{~g}$ once a day. This therapy was successful and the patient was never again afflicted with epilepsy. The patient confirmed that there had been no record of epilepsy in her previous case history.

\subsection{Surgery}

Renal transplantation was performed. The transplanted kidney secreted urine normally during establishment of its blood supply, but the postoperative urine amount gradually decreased until it reached a low of only $368 \mathrm{~mL}$ at the 14th postoperative hour. Ten hours postoperatively, reversed blood flow was observed in the transplant renal vein by color Doppler ultrasound, which suggested the presence of renal vein thrombosis (RVT). Thus, removal of the RVT was immediately implemented. After the operation, the patient was treated with urokinase thrombolysis and heparin anticoagulant therapy. As a result, transplant renal function rapidly returned to normal with urine volume maintaining a level of 4000 to $5000 \mathrm{~mL} / \mathrm{d}$ and no worsening of the creatinine level. The patient was not disturbed and experienced no discomfort.

\subsection{The patient's postoperative condition}

\subsubsection{Conditions on postoperative days 3 and 4}

During these 2 days, the general condition of the patient was well and she was able to perform the $\mathrm{Q} \& \mathrm{~A}$. With the exception of pain associated with the surgical incision, the patient reported no discomfort. Urine volume was normal, being maintained at approximately $5000 \mathrm{~mL} / \mathrm{d}$. The heparin anticoagulant therapy was performed with a continuous infusion of liquaemin for $24 \mathrm{~h}$. On the fourth postoperative day, anticoagulation therapy was stopped based on PT and APTT levels, and conventional postoperative antirejection therapy was implemented.

The application of immunosuppressants: Clinical use immunosuppressive often need to use a combination of, in order to improve the treatment effect, at the same time could reduce harmful side effects. Currently renal transplantation is relatively commonly used combination for: cyclopsorin or tacrolimus + azathioprine or? For Macaulay phenolic ester + laser

Meat.this one IST tacrolimus (FK506) + mycophenolate mofetil (MMF) + prednisone was implemented postoperatively. Tacrolimus (FK506) is from streptomycestsknbaenisis actinomycete glycolysis extracted a productKind of 23 ring big ring lactone antibiotics, 
which has strong immunosuppression function, its strength is about the meal A ring spore 50 to 100 times. mouthTake quickly absorb, main absorption parts in small intestine, absorption process similar ring spore meal A. Blood drug peak concentration appeared in oral within $0.5 \sim 3$ Hours, half-life 3.5 to 40.5 hours, average 8.7 hours, mainly by liver P4503A cell metabolism, by bravery pigment systemJuice and urinary excretion. Mainly by inhibiting intracellular calcium and calcium calmodulin and dependence on serine/threonine phosphatase neural calcium protein(the activation, blocking calcineurin) IL - 2 gene transcription, inhibiting cell activation. Oral starting dose for $0.1 \sim 0.3 \mathrm{mg} / \mathrm{kg} \mathrm{d}$.), then according to the blood drug concentration to make adjustments. Valley value concentration 1 months for $8 \sim 12 \mathrm{ng} / \mathrm{ml}$, Within $6 \sim 8 \mathrm{ng} / \mathrm{ml}$, later maintain in $4 \sim 6 \mathrm{ng} / \mathrm{ml}$ above. FK506 common side effects have diabetes, neurological side effects (including tremor, insomnia, limb abnormalities, etc), and kidney Toxicity, gastrointestinal reaction. MMF oral after being absorbed, quickly, being exactly convert bioactive Mycophenolate (MPA), plasma can't detect MMF, average oral bioavailability nearly $94 \%$, MPA in liver be metabolized to MPAG rkatsiteli grapes (MPA), renal excretion through, MPA half-life nearly 18 hours. MPA is single phosphate hypoxanthine dehydrogenase (IMPDH) reversible, a non-competitive inhibitors, restrain guanine nucleotide classic synthesis methods, lymphocyte proliferation is blocked in the cell cycle $S$ period, thus giving full play of lymphocyte immunosuppression effect. MMF Aza alternative medicine as often and ring spore meal A or tacrolimus, corticosteroids, dose doxycline for $0.5 \mathrm{~g} \sim 1.0 \mathrm{~g} /$ times every oral 2 times. MMF major adverse reaction was gastrointestinal reaction and hematopoietic system of toxic (leukopenia, thrombocytopenia).

\subsubsection{Conditions on postoperative days 5 to 11}

At 4:30 a.m. on the fifth day, the patient experienced acute paroxysmal convulsions of her lower right extremity with no obvious precipitating factor; the convulsions subsequently spread to both upper and lower extremities. Her entire body was afflicted with persistent tonic convulsions and she experienced loss of consciousness, expressionless eyes, frothing at the mouth, and gatism, all of which lasted approximately $2 \mathrm{~min}$. The patient was given a pressure pad to protect her tongue and was treated with intravenous and luminal intramuscular injections of diazepam and oral carbamazepine, which had no obvious effect. The patient experienced the aforementioned typical signs of epilepsy at intervals ranging from $30 \mathrm{~min}$ to $2 \mathrm{~h}$. These typical epileptic seizures occurred 10 times in $24 \mathrm{~h}$. Given the frequency of status epilepticus and the unusually severe drug effect, the patient underwent a neurological consultation and was treated with a continuous intravenous drip of $500 \mathrm{~mL}$ $5 \%$ glucose $+100 \mathrm{mg}$ diazepam at $10 \mathrm{~mL} / \mathrm{h}$. The patient fell into somnolence and her symptoms improved. During treatment with diazepam, although no typical epileptic attacks occurred, atypical epileptic seizures occurred 10 times in the form of twitching of the corners of the mouth and right upper extremity. On the sixth postoperative day, there were still no typical epileptic attacks with continuous use of diazepam. With a decreasing dose of diazepam, the patient was in a superficial coma, afflicted with hypomyotonia, and had an inability to move the right side of her body. A head CT scan revealed a nodule with an unclear outline in the left frontal lobe, around which patches of edema were observed. Patches of edema were also observed in the right semioval center, and the left tricorn was narrowed by the pressure. The midline shifted toward the left, resulting in edema of the brain tissue.( Fig1.2.3) 
The patient's tranquilization was continued, and she was treated for dehydration and diuresis and given supportive therapy for the right side of her body. From the seventh postoperative day, the patient was in a lighter comatose state but was afflicted with type 1 respiratory failure and pulmonary infection. Thus, she was given a breathing machine and anti-infective treatment.

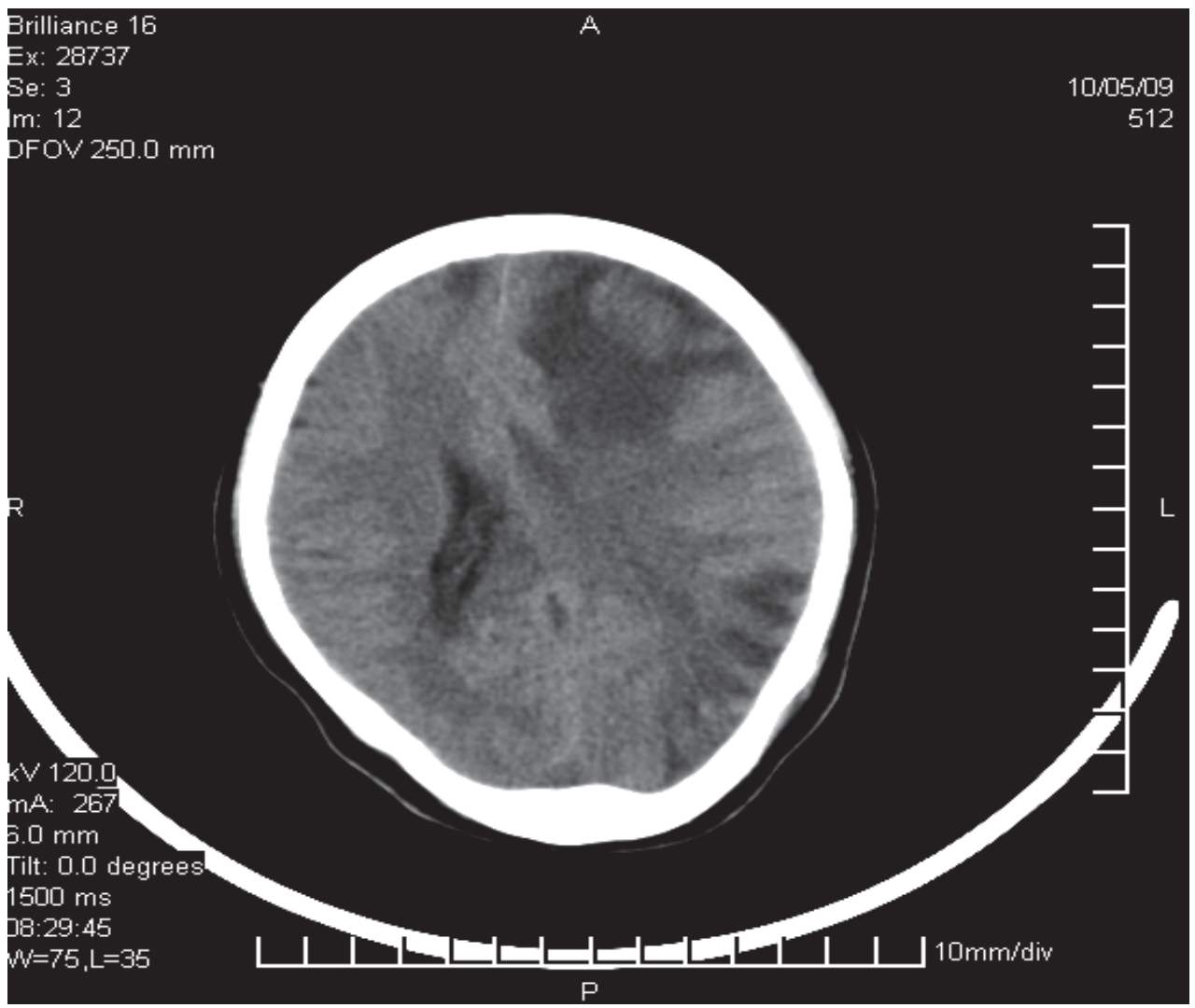

Fig 1. 


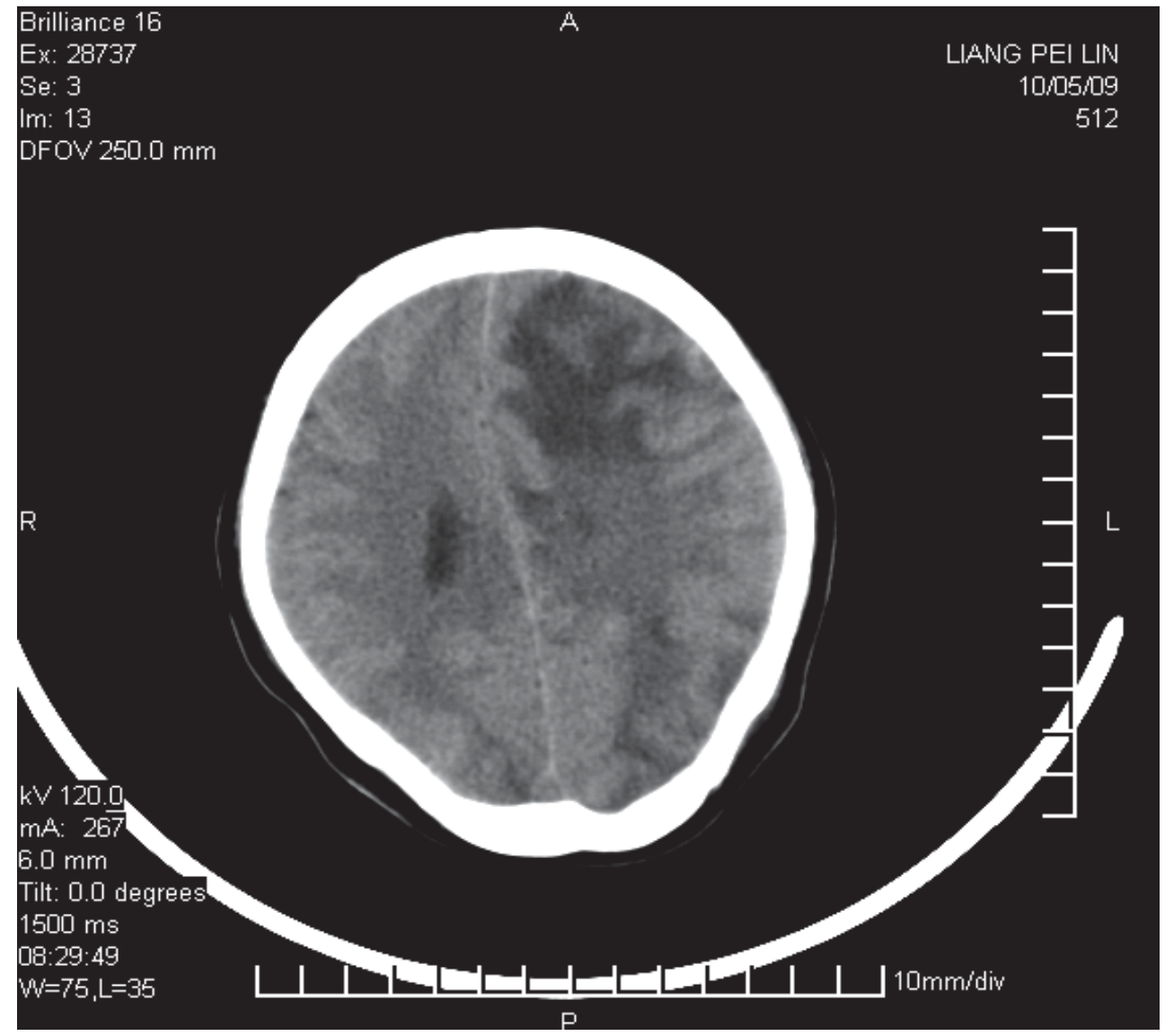

Fig. 2. 


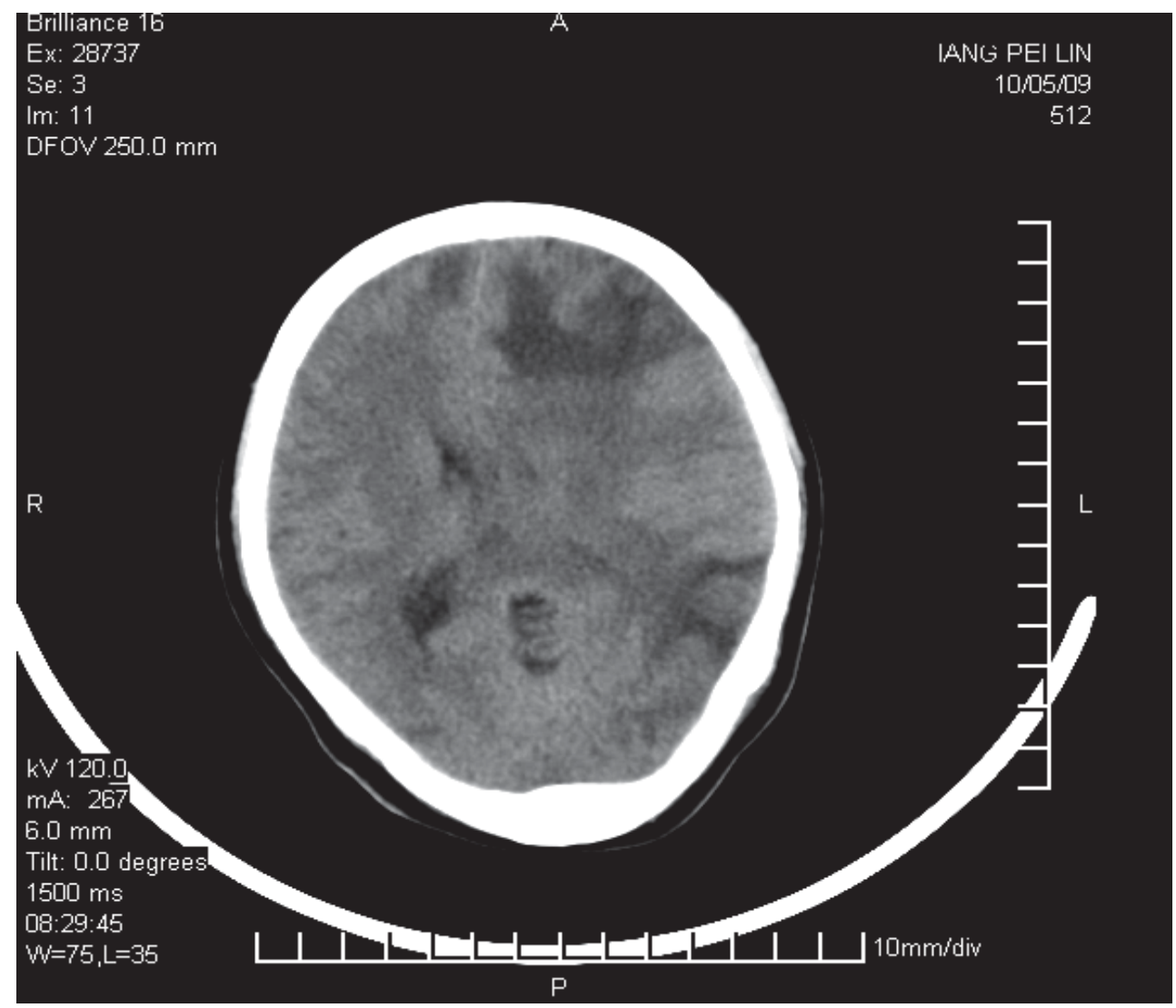

Fig. 3.

\subsubsection{Conditions on postoperative days 12 to $\mathbf{3 0}$.}

On the 12th postoperative day, the patient's condition improved with a gradual recovery of consciousness and myodynamia of the extremities. The muscular tension level of the left extremities was low, while that of the right was zero. On the 15th postoperative day, the breathing machine was removed; the muscular tension level of the left extremities returned to normal, and that of the right was five. Head CT revealed large patches of low-density areas in the semioval center of the left frontal lobe, and the left ventricle was under a small amount of pressure(Fig4.5).The patient was able to perform early ambulation, but discordance of the right extremities was still observed. Head CT on the 27th postoperative day revealed the persistence of irregular low-density areas in the left frontal lobe, and the left frontal angle had moved back slightly. With normal movement of the extremities, an almost-normal muscular tension level of the left extremities, and elimination of the pulmonary infection, the patient was cured and discharged from the hospital. 


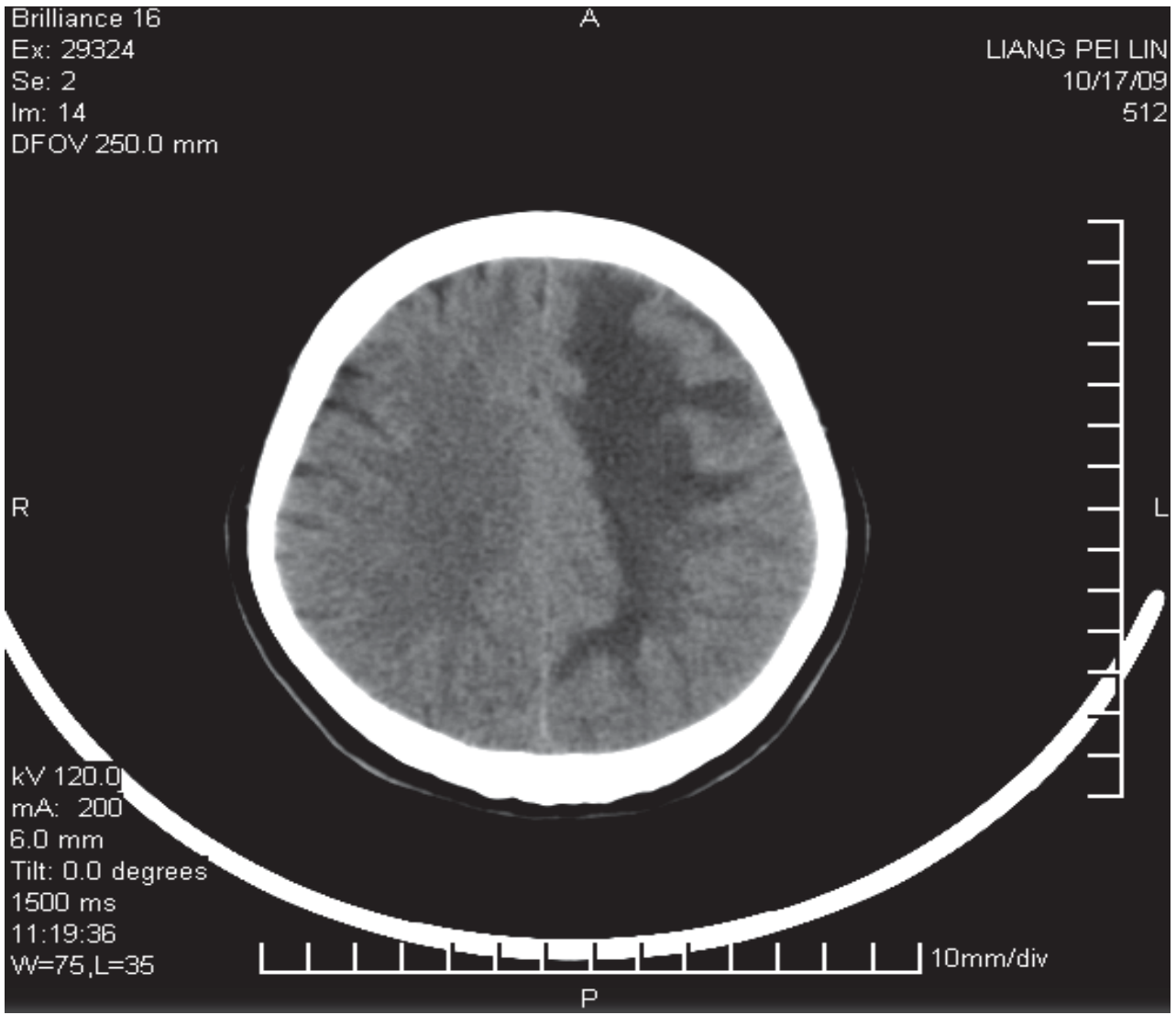

Fig. 4. 


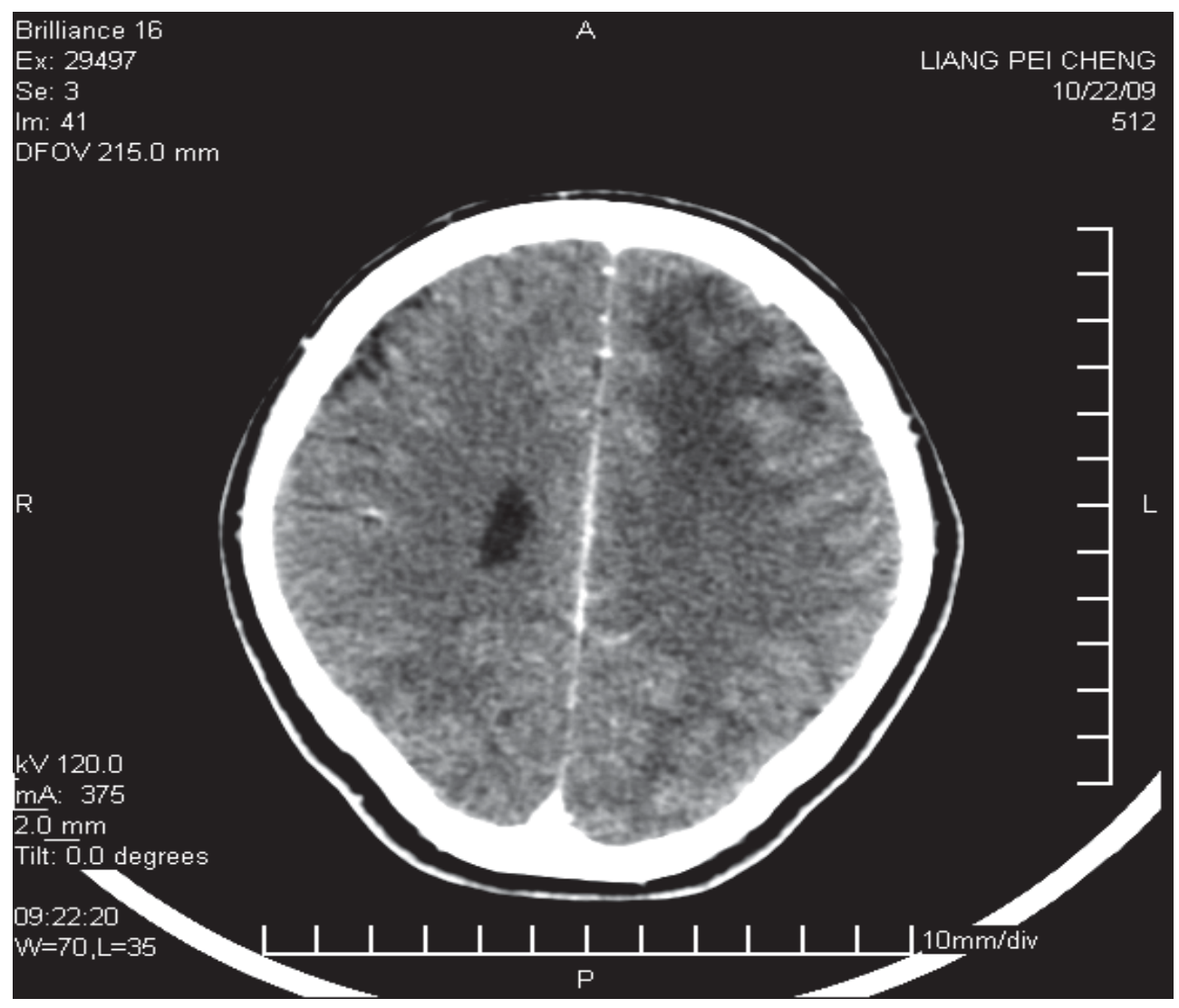

Fig. 5.

\subsubsection{Follow-ups from hospital discharge to present.}

At present, the general health condition, spirit, and appetite are good; the transplant kidney functions well; no neurological sequelae remain; and no abnormalities exist on head CT.

\subsection{The application of immunosuppressive agents.}

One aliquot of rabbit antihuman thymocyte globulin was used on the day of the operation, and another was used the following day. During the operation, $350 \mathrm{mg}$ methylprednisolone was administered via intravenous injection, and in the following 3 days, the use of methylprednisolone was continued at a dosage of $350 \mathrm{mg}$ in the first 2 days and $200 \mathrm{mg}$ on the third day. IST tacrolimus (FK506) + mycophenolate mofetil (MMF) + prednisone was implemented postoperatively. Oral administration of FK506 at a dosage of 5 to $7 \mathrm{mg} / \mathrm{kg} / \mathrm{d}$ and MMF at a dosage of $0.5 \mathrm{mg}$ twice daily was begun one day preoperatively. Because of carbamazepine's effect on FK506, the amount of FK506 was increased to achieve the target concentration. 


\subsection{Others Case report form view}

\section{Germany T. Manz report:}

A 56-year-old man with end-stage renal disease due to autosomal dominant polycystic kidney disease had continuous ambulatory peritoneal dialysis for 2 years and intermittent haemodialysis for an additional 5 years. In March 1998 his 49-year-old wife donated a kidney to him for renal transplantation. Immunosuppressive treatment consisted of prednisone, antithymocyte globulin, cyclosporin, and mycophenolate mofetil. There was delayed recovery of transplant function because of cyclosporin toxicity 1 month after transplantation. Cyclosporin dosage was reduced and mycophenolate dosage was tapered to $2 \mathrm{~g}$ /day for 1 month. Four months after transplantation cyclosporin was replaced by tacrolimus because of gingival hyperplasia and a raised serum creatinine. The blood levels of cyclosporin and tacrolimus were within therapeutic ranges. The patient's course was unremarkable until 6 months post-transplantation, when his condition deteriorated progressively, and was complicated by drowsiness, headache, impaired co-ordination and speech impediment. Axial T2 and post-contrast T1 MRI scans (Figure 1†) showed multiple 'target'-appearing ring enhancing masses in the right basal ganglia with slightly perifocal vasogenic oedema, and left frontal subcortical with central hypodensity consistent with necrosis.

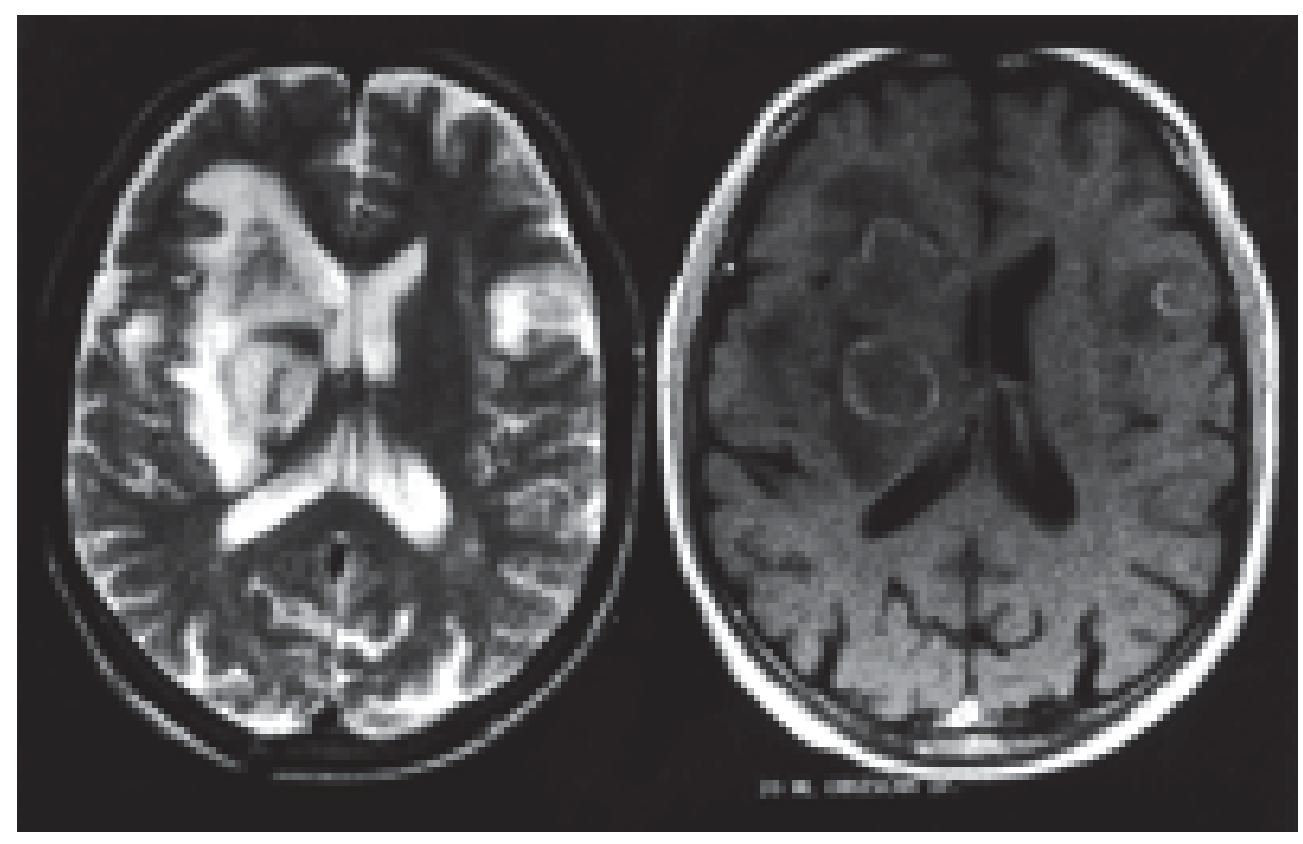

Fig. 6. Axial T2 and post-contrast T1 MR scans on admission show multiple 'target'appearing ring enhancing masses in right basal ganglia with perifocal vasogenic oedema, and left frontal subcortical with central hypodensity consistent with necrosis. 


\section{London 's Marjan Chegounchi also report:}

A 42 years old man of mixed race with an African father had originally presented with severe hypertension and renal failure. Renal biopsy showed severe vascular pathology but no primary glomerular disease. He underwent a cadaver renal transplant (111 mis-match) in July 2002 and received tacrolimus and prednisolone. The kidney functioned immediately. On day 8 (d8) with a plasma creatinine that had not fallen below $180 \mu \mathrm{mol} / 1$ and tacrolimus concentration consistently $15 \mathrm{ng} / \mathrm{ml}$ (target concentration 10-15 ng/ml), he had a renal biopsy that showed acute rejection (Banff IIa). He received intravenous methylprednisolone and Cellcept $500 \mathrm{mg}$ bd was added. The creatinine fell to125 $\mu \mathrm{mol} / 1$.

Eight weeks after transplantation (d59) he was admitted to another hospital following a road traffic accident. Whilst driving he had experienced sudden onset of drowsiness with headache and numbness in his fingers and toes. He temporarily lost consciousness and collided with another vehicle. Computed tomography (CT) scan of his head showed no abnormality and he was discharged home the following day. The following week he was readmitted (d66). He had a month history of persistent frontal headache, relieved by simple analgesic. He was intermittently confused and unable to recognize members of his family. This was associated with unsteady gait and slurred speech. During these episodes he appeared withdrawn and somewhat blank. Episodes lasted 1-8 hours. He had had one grand mal convulsion witnessed at home. There was no previous history or family history of neurological disease. On admission, he was afebrile with blood pressure of 120/75. He had no focal neurological deficit and no meningism. Cerebrospinal fluid (CSF) examination was normal: microscopy, Gram and Zeihl-Nielson stain, cytology, virology, glucose, protein, cultures and India-ink for cryptococcus were unremarkable. Blood cultures, blood count, Chest and skull X-ray were normal. Electroencephalogram (EEG) revealed sharpening and spikes independently in both temporal lobes consistent with temporal lobe partial epilepsy. The magnetic resonance imaging (MRI) of brain showed multiple areas of low signal intensity (T1-weighted FLASH) in the pons, medulla oblongata, basal ganglia and also in the cerebral hemispheres (figure 1). These lesions were reported as 'consistent with small vessel ischemia secondary to hypertension'. The diagnosis of partial epilepsy was made and he was started on lamotrigine. His renal function remained stable. Magnesium was consistently at the lower limit of normal range $0.6 \mathrm{mmol} / 1$ (normal range $0.6-1.1 \mathrm{mmol} / \mathrm{l}$ ), cholesterol $4.5 \mathrm{mmol} / 1$ (2.3 - $5.2 \mathrm{mmol} / \mathrm{l}$ ). The tacrolimus level was kept within range $8-15 \mathrm{ng} / \mathrm{dl}$.

He was readmitted three weeks later (d113) having had further fits. He was agitated, psychotic with visual hallucinations. No focal neurology was found and he rapidly became obtunded with a Glasgow coma score of 6-8/15. Repeat CT and MRI were unchanged. Lumbar puncture revealed high open pressure and raised protein $1.3 \mathrm{~g} / 1$ but CSF analysis was otherwise normal. Repeat EEG was consisted with complex partial status epileptics with "frequent frontal notched theta activity". He was treated with antibiotic and antifungal agents and phenytoin infusion. There was no improvement in his condition and a diagnosis of tacrolimus-induced neurotoxicity was made (tacrolimus $10 \mathrm{ng} / \mathrm{ml}$ ). Tacrolimus was switched to cyclosporine. Over the next few days he showed rapid and progressive improvement. He recovered fully and was discharge home. He has had no further seizure activity nor neurological symptoms since tacrolimus had been stopped. He continues to do well. A MRI scan performed in 2004 no longer showed the earlier lesions. 


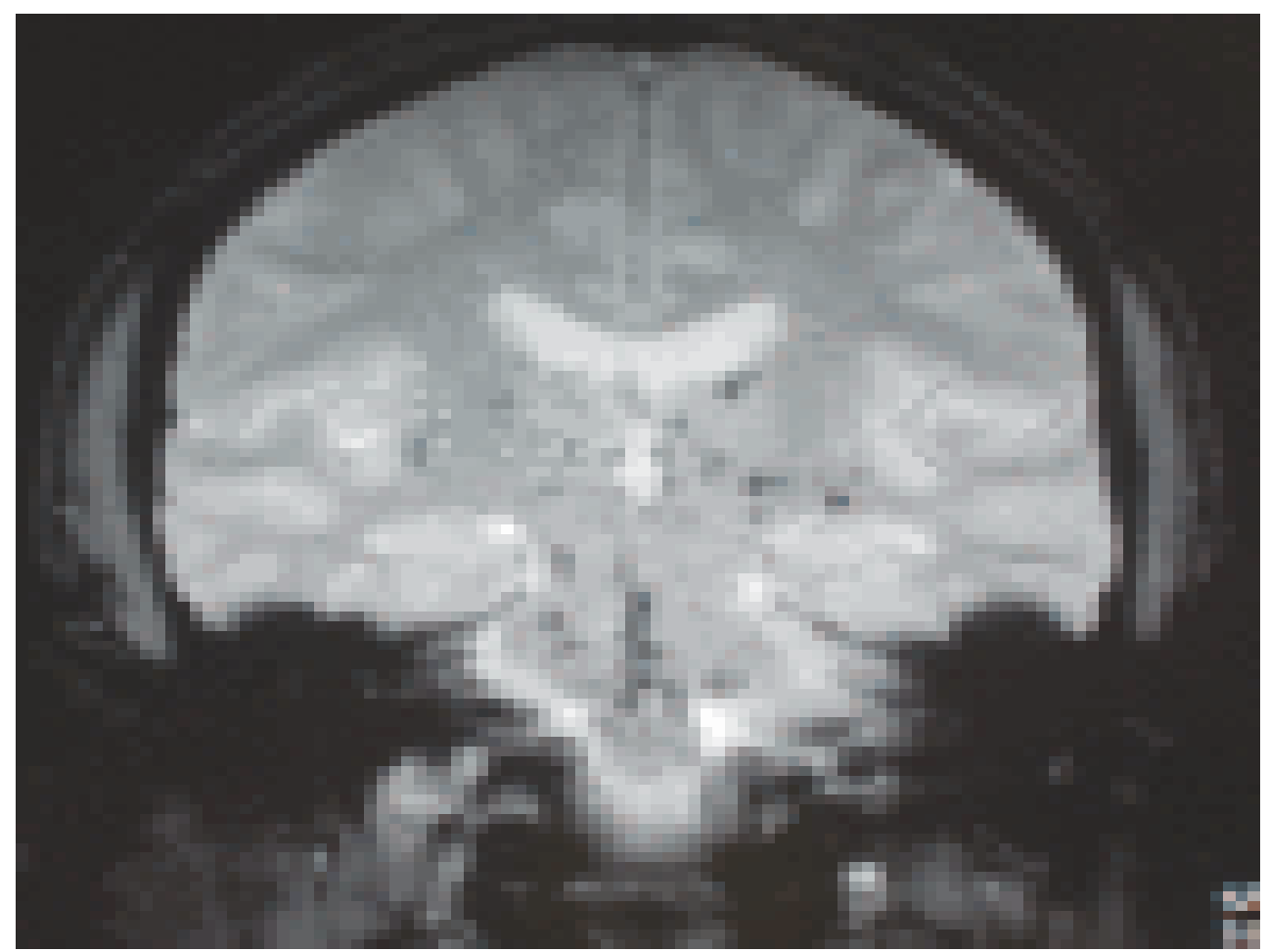

Fig. 7. MRI Brain. There are multiple areas of low signal intensity in the pons, medulla oblongata, basal ganglia and also in the cerebral hemispheres (MRI Brain T1).

\section{Discussion}

Status epilepticus is a pathological condition characterized by continuous and frequent seizures and is classified as a neurological emergency. According to conventional standards, status epilepticus consists of attacks lasting more than 30 min or repeated attacks during which consciousness is lost. New diagnostic criteria have recently been proposed by Lowenstein and other experts: ${ }^{1}$ among adults and children older than 5 years of age, generalized convulsive status epilepticus refers to a continuous seizure of more than 5 minutes in duration or more than 2 seizures at a time; during the seizure, the patient's consciousness is lost. A common clinical, and the most dangerous, form is the persistent state of the generalized tonic-clonic seizure. Symptoms are a sudden loss of consciousness, muscle twitching, foaming (sometimes with blood) at the mouth, frequent apnea, cyanosis, dilated pupils, a diminishing papillary light response, and gatism. The seizure is long in duration or occurs repeatedly. If not quickly controlled, it may be life-threatening or cause perpetual brain damage. Status epilepticus is mainly caused by improper drug decrease and withdrawal, sudden changes in medication, or nonstandard times of antiepileptic treatment administration; it may also be induced by infection, mental factors, fatigue, pregnancy, drinking, and other causes. Infection, birth injury, and congenital malformation are the main causes in infancy and childhood. Common causes in young adults include traumatic brain 
injury, intracranial masses, and parasitic diseases, while stroke, brain tumors, trauma, and degenerative diseases are the main causes in the elderly.

Nilgul Yardimci 's report: they evaluated 132 patients who had undergone a renal transplant. Ninety-seven of the recipients $(73.5 \%)$ were men and $35(26.5 \%)$ were women (M:F=2.77:1). The mean age of the recipients was $34.32 / 0.90$ years (range, 18-66 years). One hundred forty-two transplants were done; 10 patients had a retransplant. Among these 142 transplants, 36 grafts (25\%) were taken from a deceased donor, and 106 (75\%) were obtained from a living donor (deceased:living donor=1:3).

The most common renal diseases leading to renal transplant were hypertension (19 patients, $14.4 \%)$, vesicoureteral reflux (15 patients, $11.4 \%)$, and glomerulonephritis (membranoproliferative, 7 patients [5.3\%]; mesangioproliferative, 6 patients $[4.5 \%]$, and focal segmental glomerulosclerosis, 10 patients [7.6\%]). In 28 patients (21.2\%), the cause of end-stage renal disease was unknown. The mean duration of renal disease before renal transplant was 63.11 ?5.74 months (range, 1-444 months); of these, 115 had received dialysis for a mean of 35.57 ?3.24 months (range, 1-156 months). Thirty-five patients (26.5\%) had received continuous ambulatory peritoneal dialysis for a mean of 24.68 ?3.6 months (range, 1-84 months; total, 863.96 months); 89 patients $(67.4 \%)$ had undergone hemodialysis for a mean of 36.26 ?3.70 months (range, 1-144 months; total, 3227.72 months). Seventeen patients had not received any type of dialysis. At the time of transplant, 103 patients $(78 \%)$ had hypertension, and 21 (15.9\%) had hyperlipidemia. The mean follow-up was 17.26 ?0.89 months (range, 2 weeks to 40 months; total, 2279.50 months).

Neurologic complications were observed in 18 patients (13.6\%; mean age, 33.83 ?2.37 years; range, 20-59 years). Those 18 individuals experienced a total of 20 episodes of neurologic complications (2 separate episodes in 2 of the patients). The observed 20 neurologic complications were classified as follows: headache (10 episodes; $55.6 \%)$, seizure ( 3 episodes; $16.7 \%$ ), cerebral infarcts ( 2 episodes; $11.1 \%$ ), tremor ( 2 episodes; $11.1 \%$ ), encephalopathy (1 episode; $5.6 \%$ ), sinus thrombosis (1 episode; 5.6\%), and posterior leukoencephalopathy syndrome ( 1 episode, $5.6 \%$ ). Immunosuppressive agents were the primary cause of 16 of the 20 episodes of neurologic complications. Encephalopathy due to hyponatremia (1 patient), sinus thrombosis ( 1 patient), and stroke ( 2 patients) were the other causes of neurologic complications.

Of the 10 episodes with headache, 7 patients had been receiving cyclosporine for a mean of 6.04 ?2.99 months (range, 2 days to 23 months), 2 patients had been receiving sirolimus for a mean of 10.5 ?6.5 months (range, 4-17 months), and 1 patient had been receiving tacrolimus for 7 months. When cyclosporine blood levels of these patients with headache were analyzed, cyclosporine concentrations were within normal limits in 4 patients, and they were higher than normal in 3 patients. Tacrolimus and sirolimus blood concentrations all were within normal limits in the other patients with headache.

observed seizures in 3 patients, each of whom had been receiving cyclosporine, tacrolimus, or sirolimus. None of the patients had seizures before the transplant. The first patient had a seizure during the first month after transplant, and the blood concentration of cyclosporine was $305 \mathrm{ng} / \mathrm{mL}$. The second patient had been taking tacrolimus for $1 \mathrm{month}$; the blood level at the time seizure occurred was $19 \mathrm{ng} / \mathrm{mL}$. The third patient had been taking sirolimus for 3 months; the concentration at the time of the symptom was $17 \mathrm{ng} / \mathrm{mL}$. At the time of the seizure, there were no electrolyte disturbances or clinical events that could precipitate seizures. Functioning of the kidneys was within normal limits. Plasma sodium and magnesium concentrations were within normal ranges. None of these patients had a fever, 
and none was on any other medication that could cause a convulsion. The results of brain magnetic resonance imaging scans all were normal, with no evidence of a mass lesion.

Tremor developed in 2 patients who had been taking cyclosporine for a mean of $0.62 / 0.37$ months (range, 1 week to 1 month). The cyclosporine blood concentration was within normal limits in 1 patient and higher than normal in the other. However, tremor was so severe in these patients, that cyclosporine had to be switched to another calcineurin inhibitor. One patient developed posterior leukoencephalopathy syndrome after taking tacrolimus for 11 months (blood concentration, $5.5 \mathrm{ng} / \mathrm{mL}$ ). The patient clinically presented with altered mental functioning and seizures associated with symmetrical, posterior, hemispheric edema, which was apparend on cranial magnetic resonance imaging.

We observed encephalopathy due to hyponatremia $(118 \mathrm{mmol} / \mathrm{L})$ in 1 patient. Cerebrovascular infarcts were present in 2 patients, and a sinus thrombosis was observed in 1 patient.

Neural complications in renal transplant recipients are likely to occur at any stage of the postoperative period and have an incidence of $30 \%$ to $60 \%$. Both the neural complication incidence and the mortality have a great effect on renal transplant recipients. ${ }^{2-4}$ The characteristics of neural complications caused by renal transplants are distinct from those caused by other organ transplants, and include limb tremors, insomnia, dysphoria, coma, and convulsions. Epilepsy also occurs among renal transplant recipients, ${ }^{5}$ and the incidences among adults and children are $11.4 \%$ and $17.6 \%$, respectively. ${ }^{6}$ In cats with end-stage renal failure that have undergone renal transplants, the incidence of epilepsy may reach $28.9 \% .{ }^{7}$

Many factors can lead to epilepsy after transplantation, among which electrolyte disturbance is the most common. ${ }^{8}$ Characteristics include early epileptic attacks, usually in the 72 nd postoperative hour; a high urine volume at $10000 \mathrm{~mL} / \mathrm{d}$; rapid recovery of renal function; and the return of SCr to a normal level at the first epileptic attack. Epilepsy can be easily controlled by correction of electrolyte disturbances with supplementation of blood calcium, magnesium, and sodium and intramuscular injections of diazepam. However, the causes of status epilepticus might be linked to withdrawal of antiepileptic drugs (AEDs) and intoxication $^{9}$ of the calcineurin inhibitors cyclosporine and tacrolimus. Epilepsy can be caused by intoxication of cyclosporine and tacrolimus, ${ }^{10}$ the incidences of which are $2 \%$ to $6 \% 11-14$ and $5.6 \%$ to $11.6 \% .{ }^{15}$ Antirejection therapy can be impacted by the use of larger doses of hormones, by which the patient's mental state can be changed and epilepsy can be easily triggered. This type of epilepsy has more severe symptoms, easily causes acute respiratory distress syndrome, and maintains a high mortality; it is also very difficult to cure, often requiring tracheal cannulation and positive end-expiratory pressure with a breathing machine. The aforementioned patient was afflicted by epilepsy once during the month before the transplant. Because of AED withdrawal, the impact of MMP, and the use of tacrolimus, children and young adults with a history of epilepsy are afflicted with epilepsy more frequently, and its persistent state is caused by neurological diseases under the combined action of hormones and physiological changes. It was found by Gleeson through 3-year follow-ups of patients with epileptic seizures during the perioperative period during AED withdrawal, the patients were never afflicted with epilepsy. ${ }^{16}$

Many renal transplant patients have some degree of vascular compromise, either as a result of their underlying disease (hypertension, diabetes) or because of emboli associated with underlying atherosclerosis or heart disease (5). The most common posttransplant neurologic complications in this patient population are cerebrovascular events; these occur in 
approximately $9 \%$ of all renal transplant patients. infarcts in 2 patients (1.5\%). Stroke may occur in about $8 \%$ of renal transplant patients.

An increased risk of venous thromboembolism also has been demonstrated after renal transplant. Commonly reported sites have been deep vein thrombosis, pulmonary thromboembolism, and vascular thrombosis involving the graft. Cerebral venous thrombosis has not been reported thus far. assessed transverse sinus thrombosis in 1 patient $(0.8 \%)$.Tremor is another common complication, which is frequently seen after transplant; sometimes present in up to $40 \%$ of patients. Tremor is mostly the consequence of immunosuppressive treatment. The most pronounced neurotoxic effect is induced by the calcineurin inhibitors, tacrolimus and cyclosporine. The spectrum of neurologic disturbances caused by calcineurin inhibitors ranges from mild symptoms such as paraesthesia, tremor, headache, or flushing, to severe changes that can cause a lethal outcome. In our study, we evaluated 2 patients $(1.5 \%)$ with tremor while taking cyclosporine. To control tremor, the drug must be switched to another calcineurin inhibitor. In fact, tremor in most of the recipients is the result of immunosuppressive agents. However, if severity of the tremor is not significant and does not worsen the patient's quality of life, there is a tendency to ignore the symptom (because survival of the graft is the ultimate goal in immunosuppressive therapy). In the current study, we observed only 2 tremors; however, these were severe enough to warrant a change in the immunosuppressive protocol. The slight tremor in the other recipients probably had been underestimated in the patients' records.Posterior leukoencephalopathy syndrome is most commonly seen in patients with hypertensive encephalopathy, eclampsia, renal failure, or use of immunosuppressive agents (20). In the transplant population, posterior leukoencephalopathy synrome is a well-known complication of immunouppressive therapy with cyclosporine and tacrolimus. In the current study, we observed 1 patient $(0.8 \%)$ with posterior leukoencephalopathy syndrome. Posterior leukoencephalopathy syndrome is a term first used by Hinchey and associates (20) to describe a group of disorders that present clinically with headache, seizures, visual disturbances, and altered mental function associated with symmetrical posterior hemispheric edema. The cause of posterior leukoencephalopathy syndrome is not fully undertood but is believed to be due to a breakdown in cerebral autoregulation that results in leakage of fluid into the interstitium, which is detected as vasogenic edema. (21) Encephalopathy is a severe adverse effect of cyclosporine and tacrolimus, occurring in approximately $5 \%$ of patients taking the drugs. These patients may present with a decreased level of consciousness, headache, dysarthria, depression, mania, cortical blindness, visual hallucinations, and seizures . (22)The syndrome is usually found in patients with elevated blood calcineurin inhibitor levels; however, other factors, such as hypoholesterolemia, hypomagnesemia, hypoatremia, high-dose steroids, hypertension, and uremia may be involved as well. We observed encephalopathy due to hyponatremia in 1 patient $(0.8 \%)$; the encephalopathy resolved after the hyponatremia ameliorated. In the current study, we did not evaluate any malignancies or central nervous system infections that are other frequent causes of neurologic complications in transplant recipients.

The grand mal seizures after renal transplantation were usually serious. However, the patient was cured without neurological sequelae, and the transplant kidney recovered very well. Experience with this diagnosis and treatment regimen has been gained in the following 4 respects. First, while the patient's life must be saved, transplant renal function must be maintained and delayed graft function (DGF) must be prevented, which is the core 
of successful treatment. Changes in the patient's blood pressure and heart rate can be caused by epileptic seizures. In particular, status epilepticus has a more serious effect on the circulatory system, easily triggering DGF because of the adverse effect on transplant renal function. Once DGF has been triggered, the whole treatment will be more difficult. Second, accurate judgment of the causes of epilepsy is critical to effective diagnosis and treatment. Different causes, such as brain tumors, cerebrovascular accident, and drug-induced factors, require different treatments. However, the treatment should be simplified in case the patient's condition is complicated. Third, when epilepsy occurs during the perioperative period, immunosuppressive agents ${ }^{17,} 18$ should be appropriately adjusted. Because of the interaction between AEDs and antirejection drugs, blood concentrations of cyclosporine and tacrolimus can be dramatically lowered by the carbamazepine. Thus, blood concentrations of carbamazepine and tacrolimus should be monitored so that the adjustment of immunosuppressive agents can be performed in time. Finally, comprehensive measures should be taken to prevent other complications and reduce adverse effects in the therapeutic process. Aspiration can easily be caused by seizures, and status epilepticus requires patients to stay in bed; both increase the possibility of pulmonary infection. In addition, central respiratory problems can be caused by severe cerebral problems. The importance of prevention and cure of pulmonary infection is determined by the complexity, refractory characteristics, and high mortality of pulmonary infection after transplant.

The improvement of surgical techniques of organ transplantation and specific nursing practices that can reduce mortality and morbidity make it possible to perform many organ transplantations. The postoperative period of organ transplantation mostly focuses on infection prevention and antirejection treatment. Most neural complications are atypical and have not been given enough attention. However, a certain amount of morbidity and mortality is still attributed to serious neural complications. On one hand, neural complications in some renal transplant recipients are caused by postoperative diseases. On the other hand, neurotoxicity caused by immunotherapy medication can also lead to a series of complications. The adverse effects of the immune overreaction to cyclosporine and tacrolimus should be noted. ${ }^{19}$ Many factors, acting alone or in combination, can cause epileptic seizures after renal transplantation. Thus, for prevention and cure, great importance should be attached to comprehensive treatment. Drugs that can trigger epileptic seizures should be avoided. If epileptic seizures take place, the pathogenesis should be determined and symptomatic therapy begun.

\section{References}

[1] Lowenstein DH, Bleck TP, Macdonald RL. It's time to revise the defi-nition of status epilepticus. Epilepsia, 1999, 40 (1) : 120 - 122

[2] Bruno A, Adams HP Jr. Neurologic problems in renal transplant recipients. Neurol Clin. 1988;6:305-325.

[3] Mart ez AJ. The neuropathology of organ transplantation:comparison and contrast in 500 patients. Pathol Res Pract. 1998; 194:473-486.

[4] Schwechheimer K, Hashemian A. Neuropathologic findings after organ transplantation. An autopsy study. Gen Diagn Pathol. 1995;141:35-39.

[5] Hamiwka LD, Midgley JP, Hamiwka LA.Seizures in children after kidney transplantation: has the risk changed and can we predict who is at greatest risk? Pediatr Transplant. 2008 Aug;12(5):527-30. 
[6] Awan A Q , Lewis MA , Postleth waite R J ,et al. Seizures fol-lowing renal transplantation in childhood. Pediatr-Nephrol , 1999, 13:275 - 279.

[7] Mathews K G, Gregory C R. Renal transplants in cats :66 cas-es. J Am Vet Med Assoc, $1997,211: 1432$ - 1436.

[8] Wan Jun R., Zi Dong L, Xiao Ping W. 2 Cases of Epilepsy Tonic - Clonic Seizures after Renal Transplantation. Chinese Journal of Organ Transplantation.2002;23:235 - 235

[9] Patchell RA. Neurological complications of organ transplantation. Ann Neurol. 1994;36:688-703

[10] Appignani BA, Bhadelia RA, Blacklow SC, Wang AK, Roland SF, Freeman RBJ: Neuroimaging findings in patients on immunosuppressive therapy: experience with tacrolimus toxicity. AJR Am J Roentgenol 1996, 166:683-688.

[11] Racusen LC, Famiglio LM, Fivush BA, Olton DS, Solez K. Neurologic abnormalities and mortality in rats treated with cyclosporine A. Transplant Proc. 1988;20(suppl 3):934-936.

[12] Graham RM. Cyclosporine: mechanisms of action and toxicity. Cleve Clin J Med. 1994; 61:308-313.

[13] Gijtenbeek JM, van den Bent MJ, Vecht CJ. Cyclosporine neurotoxicity: a review. J Neurol. 1999;246:339-346.

[14] Walker RW, Brochstein JA. Neurologic complications of immunosuppressive agents. Neurol Clin. 1988;6:261-278

[15] Sevmis S, Karakayali H, Emiroglu R, Akkoc H, Haberal M. Tacrolimus-related seizure in the early postoperative period after liver transplantation. Transplant Proc. 2007;39:1211-12

[16] Gleeson JG, duPlessis AJ, Barnes PD, Riviello JJJ.Cyclosporin A acute encephalopathy and seizure syndrome in childhood: clinical features and risk of seizure recurrence. J Child Neurol 1998, 13:336-344

[17] Yardimci N, Colak T, Sevmis S, Benli S .Neurologic complications after renal transplant Exp Clin Transplant. 2008 Sep;6(3):224-8..

[18] Senzolo M, Ferronato C, Burra P.Neurologic complications after solid organ transplantation. Transpl Int. 2009 Mar;22(3):269-78.

[19] Yardimci N, Colak T, Sevmis S, Benli S.Neurologic complications after renal transplant. Exp Clin Transplant. 2008 Sep;6(3):224-8.

[20] Hinchey J, Chaves C, Appignani B, et al. A reversible posterior leukoencephalopathy syndrome. N Engl J Med. 1996;334:494-500.

[21] Mukherjee P, McKinstry RC. Reversible posterior leukoencephalopathy syndrome: evaluation with diffusion-tensor MR imaging. Radiology. 2001;219:756-765.

[22] Adams DH, Ponsford S, Gunson B, et al. Neurological complications following liver transplantation. Lancet. 1987;1:949-951. 


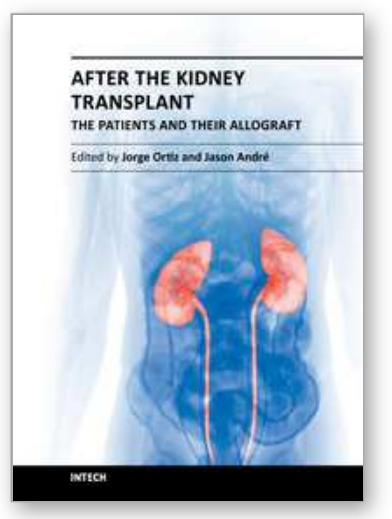

\author{
After the Kidney Transplant - The Patients and Their Allograft \\ Edited by Prof. Jorge Ortiz
}

ISBN 978-953-307-807-6

Hard cover, 386 pages

Publisher InTech

Published online 17, August, 2011

Published in print edition August, 2011

There are many obstacles in kidney transplantation. For the transplant team, there is the balance between immunosuppression to aid in the recipientâ $€^{\mathrm{TM}} \mathrm{S}$ tolerance of the allograft and the infection risk of a suppressed immune system. These potential long term complications of kidney transplantation are relatively well known, but there are many other complications that patients and families do not consider when preparing themselves for a kidney transplant. Although the benefits of attempting a kidney transplant far outweigh downfalls of the long term sequelae, kidney transplantation is by no means a benign procedure. It is the hope of these authors that the reader will leave with a sense of understanding towards the kidney recipients.

\title{
How to reference
}

In order to correctly reference this scholarly work, feel free to copy and paste the following:

Gao Hong-jun, Luo xiangdong, Liang Taisheng, Liang Fangfang et al. (2011). Diagnosis and Treatment of Status Epilepticus in a Pediatric Renal Recipient, After the Kidney Transplant - The Patients and Their Allograft, Prof. Jorge Ortiz (Ed.), ISBN: 978-953-307-807-6, InTech, Available from:

http://www.intechopen.com/books/after-the-kidney-transplant-the-patients-and-their-allograft/diagnosis-andtreatment-of-status-epilepticus-in-a-pediatric-renal-recipient

\section{INTECH}

open science | open minds

\section{InTech Europe}

University Campus STeP Ri

Slavka Krautzeka 83/A

51000 Rijeka, Croatia

Phone: +385 (51) 770447

Fax: +385 (51) 686166

www.intechopen.com

\section{InTech China}

Unit 405, Office Block, Hotel Equatorial Shanghai

No.65, Yan An Road (West), Shanghai, 200040, China 中国上海市延安西路65号上海国际贵都大饭店办公楼 405 单元

Phone: +86-21-62489820

Fax: $+86-21-62489821$ 
(C) 2011 The Author(s). Licensee IntechOpen. This chapter is distributed under the terms of the Creative Commons Attribution-NonCommercialShareAlike-3.0 License, which permits use, distribution and reproduction for non-commercial purposes, provided the original is properly cited and derivative works building on this content are distributed under the same license. 